\title{
The Effect of the Zero-Difference Policy on the Financial Situation of Public Hospitals in Different Grades \\ Chun-li LIU
}

School of management, Xinxiang Medical College. Healthy Zhongyuan Research Institute, China

Keywords: Drug price difference, Public hospitals, Grades, Finance.

Abstract. Based on the different levels of public hospitals in zero price difference before and after the visits, both times cost data were analyzed, such as study drug zero price difference influence on hospital financial situation, and improve the hospital reform of financial advice is put forward.

\section{零差价政策对不同等级公立医院财务状况的影响}

\author{
刘春利
}

新乡医学院管理学院, 健康中原研究院, 中国

关键词: 药品零差价; 公立医院; 等级; 财务

摘要: 通过对不同等级公立医院在药品零差价前后诊疗人次、均次费用等数据进行对比分析, 探讨药品零差价对医院财务状况的影响, 并提出完善医院改革的财务建议。

1. 引言

为了加快公立医院改革, 破除 “以药补医” 机制, 实现医疗技术的价值回归, 减少公立 医院的逐利行为。文章通过对 2010年至2014年期间三个等级公立医院财务数据的比较, 分析 执行药品零差率政策对其财务状况的影响，并提出完善公立医院改革的相关建议。

\section{2. 资料来源及方法}

选取公立医院医改前后医疗业务报表数据，包括医院诊疗人次、门诊均次费用、住院均 次费用等, 并把公立医院分为三个等级, 加以比较说明。采用因素分析法、比率分析法等, 利用 Excel 软件进行数据整理, 将每个指标与上年同期数据对比, 分析医院有关指标的变化 情况。（注：数据来源于2015中国卫生和计划生育统计年鉴）

\section{3. 分析与结果}

\section{1 公立医院诊疗人次变化}

从表 1 可知，医改政策实施前后这近几年，其中三级医院门急诊人次 2010 年为76046.3 万人次，2014年高达 139804.4 万人次，增幅比例为 $83.8 \%$ 。

表 2 中, 诊疗人次数增幅每年保持增长，只是幅度不同，其中三级医院在 2012 年比 2011 年大幅增长了 $21 \%$, 之后也保持 $13 \%$ 左右的增长; 三个等级中, 都是 2012 年增长最多, 之后 2 年有回落, 保持稳定。 
表1 公立医院诊疗人次数（万人次）

\begin{tabular}{cccccc}
\hline 等级 & 2010 & 2011 & 2012 & 2013 & 2014 \\
\hline 三级医院 & 76046.3 & 89807.8 & 108670.6 & 123821.9 & 139804.4 \\
二级医院 & 93120.4 & 99198.5 & 105476.7 & 109169.1 & 114708.6 \\
一级医院 & 14573.6 & 15336.5 & 16766.5 & 17617.9 & 18478.1 \\
\hline \multicolumn{5}{c}{ 表2 } & 公立医院诊疗人次数增幅\% \\
\hline 等级 & 2011 & 2012 & 2013 & 2014 \\
三级医院 & 18.1 & 21.0 & 13.9 & 12.9 \\
二级医院 & 6.5 & 6.3 & 3.5 & 5.1 \\
一级医院 & 5.2 & 9.3 & 5.1 & 4.9 \\
\hline
\end{tabular}

\section{2 患者均次费用的变化}

患者均次费用是度量病人经济负担的标尺,2010-2013 年由于受均次费用 “零增长” 政策 的约束, 各家医院采取了各种措施来控制患者均次费用, 2014 年该项控费政策放宽, 均次费 用出现 “反弹”。

\subsection{1 门诊均次费用的变化}

从表 3 可知, 医改前后门诊均次费用增长幅度降低, 说明药品零差率政策对医院门诊均 次费用的控制效果逐渐呈现。

表3 医院门诊病人次均医药费用（元）

\begin{tabular}{cccccc}
\hline \multirow{2}{*}{ 指标 } & \multicolumn{2}{c}{$\begin{array}{l}\text { 门诊病人次均 } \\
\text { 医药费 (元) }\end{array}$} & \multicolumn{2}{c}{ 其中: } & \multicolumn{2}{c}{ 占门诊医药费 } & 检查费 & 药费 & 检查费 \\
\hline 医院合计 & & & & & \\
2010 & 166.8 & 85.6 & 30.0 & 51.3 & 18.0 \\
2011 & 179.8 & 90.9 & 32.4 & 50.5 & 18.0 \\
2012 & 192.5 & 96.9 & 35.0 & 50.3 & 18.2 \\
2013 & 206.4 & 101.7 & 37.4 & 49.3 & 18.1 \\
2014 & 220.0 & 106.3 & 40.3 & 48.3 & 18.3 \\
\hline
\end{tabular}

表 4 , 其中公立医院, 指标明显高于平均值, 比如: 门诊病人次均医药费, 都偏高; 药费 占门诊医药费比例都高。说明这一部门内容在公立医院需要引起重视。

表4 医院门诊病人次均医药费用（元）

\begin{tabular}{cccccc}
\hline 指标 & $\begin{array}{l}\text { 门诊病人次均 } \\
\text { 医药费 (元) }\end{array}$ & \multicolumn{2}{c}{ 其中: } & \multicolumn{2}{c}{ 占门诊医药费 } \\
& & & & & \\
检查费 & 药费 & 检查费 \\
\hline 其中: 公立医院 & & & & & \\
2010 & 167.3 & 87.4 & 30.8 & 52.3 & 18.4 \\
2011 & 180.2 & 92.8 & 33.4 & 51.5 & 18.5 \\
2012 & 193.4 & 99.3 & 36.2 & 51.3 & 18.7 \\
2013 & 207.9 & 104.1 & 38.7 & 50.2 & 18.6 \\
2014 & 221.6 & 109.3 & 41.8 & 49.3 & 18.9 \\
\hline
\end{tabular}

由表5、表6、表7可知, 三级医院相比较于其他等级医院，门诊病人次均费用更为偏高， 2014年三级医院次均费用为 269.8 元, 比平均值 220.0 元高了近 50 元; 当然其他等级医院可能由 于服务水平差异，导致病人次均费用偏低。 
但同时发现, 表7中, 一级医院的药占比, 却是最高的, 高达 $53.0 \%$, 说明极有可能在低 等级医院存在过度依赖药品收入行为。

表5 医院门诊病人次均医药费用（元）

\begin{tabular}{|c|c|c|c|c|c|}
\hline \multirow{2}{*}{ 指标 } & \multirow{2}{*}{$\begin{array}{l}\text { 门诊病人次均 } \\
\text { 医药费 (元) }\end{array}$} & \multicolumn{2}{|c|}{ 其中: } & \multicolumn{2}{|c|}{ 占门诊医药费\% } \\
\hline & & 药费 & 检查费 & 药费 & 检查费 \\
\hline \multicolumn{6}{|l|}{ 内: 三级医院 } \\
\hline 2010 & 220.2 & 117.6 & 37.9 & 53.4 & 17.2 \\
\hline 2011 & 231.8 & 122.0 & 40.2 & 52.6 & 17.3 \\
\hline 2012 & 242.1 & 126.7 & 42.7 & 52.3 & 17.6 \\
\hline 2013 & 256.7 & 132.1 & 45.2 & 51.5 & 17.6 \\
\hline 2014 & 269.8 & 136.0 & 48.4 & 50.4 & 17.9 \\
\hline \multicolumn{6}{|c|}{ 表6 医院门诊病人次均医药费用（元） } \\
\hline \multirow{2}{*}{ 指标 } & \multirow{2}{*}{$\begin{array}{c}\text { 门诊病人次均医 } \\
\text { 药费 (元) }\end{array}$} & \multicolumn{2}{|c|}{ 其中: } & \multicolumn{2}{|c|}{ 占门诊医药费\% } \\
\hline & & 药费 & 检查费 & 药费 & 检查费 \\
\hline \multicolumn{6}{|l|}{ 二级医院 } \\
\hline 2010 & 139.3 & 70.5 & 28.9 & 50.6 & 20.8 \\
\hline 2011 & 147.6 & 73.6 & 31.0 & 49.9 & 21.0 \\
\hline 2012 & 157.4 & 77.9 & 33.3 & 49.5 & 21.1 \\
\hline 2013 & 166.2 & 79.6 & 35.2 & 47.9 & 21.2 \\
\hline 2014 & 176.0 & 82.8 & 37.7 & 47.1 & 21.4 \\
\hline \multicolumn{6}{|c|}{ 表7 医院门诊病人次均医药费用（元） } \\
\hline \multirow{2}{*}{ 指标 } & \multirow{2}{*}{$\begin{array}{l}\text { 门诊病人次均 } \\
\text { 医药费 (元) }\end{array}$} & \multicolumn{2}{|c|}{ 其中: } & \multicolumn{2}{|c|}{ 占门诊医药费\% } \\
\hline & & 药费 & 检查费 & 药费 & 检查费 \\
\hline \multicolumn{6}{|l|}{ 一级医院 } \\
\hline 2010 & 93.1 & 51.6 & 11.5 & 55.4 & 12.4 \\
\hline 2011 & 103.9 & 56.1 & 13.4 & 54.0 & 12.9 \\
\hline 2012 & 112.0 & 59.9 & 14.7 & 53.5 & 13.1 \\
\hline 2013 & 119.8 & 64.2 & 15.6 & 53.6 & 13.1 \\
\hline 2014 & 125.3 & 66.4 & 17.1 & 53.0 & 13.7 \\
\hline
\end{tabular}

\section{2 .2 住院人均费用的变化}

从表 7 可知，医改前后住院人均费用，2010年为6193.9元，2014年为7832.3元，

且增长幅度总体趋势向缓, 2011 年较 2010 年增幅为 7.1\%, 2012 年较 2011 年增幅为 $5.2 \%, 2014$ 年较 2013 年增幅为 5.2\%; 其中药费占住院医药费比例逐年降低, 2010 年 43.1\%, 2014 年为 $38.3 \%$, 说明 医改后这项控制得比较良好。

表7 医院住院病人人均医药费用（元）

\begin{tabular}{cccccc}
\hline 指标 & $\begin{array}{c}\text { 住院病人人均医药 } \\
\text { 费 (元) }\end{array}$ & \multicolumn{2}{c}{ 其中: } & \multicolumn{2}{c}{ 占住院医药费\% } \\
\hline 医院合计 & & & & & \\
2010 & 6193.9 & 2670.2 & 441.5 & 43.1 & 7.1 \\
2011 & 6632.2 & 2770.5 & 492.7 & 41.8 & 7.4 \\
2012 & 6980.4 & 2867.4 & 533.9 & 41.1 & 7.6 \\
2013 & 7442.3 & 2939.1 & 590.2 & 39.5 & 7.9 \\
2014 & 7832.3 & 2998.5 & 640.6 & 38.3 & 8.2 \\
\hline
\end{tabular}


表8＼cjkstart医院住院病人人均医药费用（元）

\begin{tabular}{cccccc}
\hline \multirow{2}{*}{ 指标 } & $\begin{array}{c}\text { 住院病人人均医药 } \\
\text { 费 (元) }\end{array}$ & \multicolumn{2}{c}{ 其中: } & \multicolumn{2}{c}{ 占住院医药费\% } \\
其中: 公立医院 & & & & & \\
2010 & 6415.9 & 2784.3 & 460.8 & 43.4 & 7.2 \\
2011 & 6909.9 & 2903.7 & 518.5 & 42.0 & 7.5 \\
2012 & 7325.1 & 3026.7 & 565.4 & 41.3 & 7.7 \\
2013 & 7858.9 & 3116.3 & 629.8 & 39.7 & 8.0 \\
2014 & 8290.5 & 3187.1 & 685.2 & 38.4 & 8.3 \\
\hline
\end{tabular}

公立医院相比较于医院平均值, 略偏高。其中表9、表10、表11, 三级医院更是与其他等 级相比, 次均费用高出很多。说明医疗资源不同, 存在分配不均。一级医院次均费用很低, 可能只能治疗一些难度不高的疾病, 但是医药费用占比却是最高的达 $40.7 \%$, 这和门诊的结果 是极为相似的。

表9＼cjkstart医院住院病人人均医药费用（元）

\begin{tabular}{|c|c|c|c|c|c|}
\hline \multirow{2}{*}{ 指标 } & \multirow{2}{*}{$\begin{array}{l}\text { 住院病人人均医 } \\
\text { 药费（元） }\end{array}$} & \multicolumn{2}{|c|}{ 其中: } & \multicolumn{2}{|c|}{ 占住院医药费\% } \\
\hline & & 药费 & 检查费 & 药费 & 检查费 \\
\hline \multicolumn{6}{|c|}{ 内: 三级医院 } \\
\hline 2010 & 10442.4 & 4440.9 & 765.5 & 42.5 & 7.3 \\
\hline 2011 & 10935.9 & 4480.4 & 838.7 & 41.0 & 7.7 \\
\hline 2012 & 11186.8 & 4521.0 & 881.1 & 40.4 & 7.9 \\
\hline 2013 & 11722.4 & 4578.3 & 952.1 & 39.1 & 8.1 \\
\hline 2014 & 12100.2 & 4610.1 & 1007.0 & 38.1 & 8.3 \\
\hline \multicolumn{6}{|c|}{ 表10 医院住院病人人均医药费用（元） } \\
\hline \multirow{2}{*}{ 指标 } & \multirow{2}{*}{$\begin{array}{c}\text { 住院病人人均医 } \\
\text { 药费（元） }\end{array}$} & \multicolumn{2}{|c|}{ 其中: } & \multicolumn{2}{|c|}{ 占住院医药费\% } \\
\hline & & 药费 & 检查费 & 药费 & 检查费 \\
\hline \multicolumn{6}{|c|}{ 二级医院 } \\
\hline 2010 & 4338.6 & 1944.8 & 303.4 & 44.8 & 7.0 \\
\hline 2011 & 4564.2 & 1999.2 & 332.3 & 43.8 & 7.3 \\
\hline 2012 & 4729.4 & 2033.3 & 352.4 & 43.0 & 7.5 \\
\hline 2013 & 4968.3 & 2028.4 & 389.0 & 40.8 & 7.8 \\
\hline 2014 & 5114.6 & 2003.9 & 417.4 & 39.2 & 8.2 \\
\hline \multicolumn{6}{|c|}{ 表11 医院住院病人人均医药费用（元） } \\
\hline \multirow{2}{*}{ 指标 } & \multirow{2}{*}{$\begin{array}{l}\text { 住院病人人均医 } \\
\text { 药费 (元) }\end{array}$} & \multicolumn{2}{|c|}{ 其中: } & \multicolumn{2}{|c|}{ 占住院医药费\% } \\
\hline & & 药费 & 检查费 & 药费 & 检查费 \\
\hline \multicolumn{6}{|c|}{ 一级医院 } \\
\hline 2010 & 2844.3 & 1243.7 & 185.9 & 43.7 & 6.5 \\
\hline 2011 & 3121.3 & 1364.4 & 207.0 & 43.7 & 6.6 \\
\hline 2012 & 3285.0 & 1411.3 & 236.1 & 43.0 & 7.2 \\
\hline 2013 & 3561.9 & 1471.2 & 277.7 & 41.3 & 7.8 \\
\hline 2014 & 3737.1 & 1519.8 & 311.5 & 40.7 & 8.3 \\
\hline
\end{tabular}




\section{4. 讨论}

通过分析可以发现, 医改之后公立医院的医疗费用结构正逐步由药品主导向技术和服务 主导转变, 药占比有所下降。取消药品加成带来的损失, 通过提高四大类医疗服务项目的价 格得到弥补, 部分技术劳务项目的价格有较大幅度的提高, 实现了一定程度的劳务价值回归, 这将有益于提高医务人员的积极性。从控制药品费用的角度来看, 实施药品零差率政策也取 得一定的积极效果。

\section{5. 建议}

\section{1 加大均次费用控制力度}

现行的药品集中招标制度, 不能充分激励医院在控制药品价格上发挥作用。因此, 尽管 实行了药品零差率销售, 医院仍没有降低药价的动力。作为药品采购最为直接的利益相关方, 如果允许医院通过 “二次谈判” 降低药品的采购价格, 激发医院降低药品费用的动力, 同时 实现 “以药养药”，维持药剂人员队伍的稳定。

\section{2 进一步调整、规范医疗服务价格体系}

本轮医改提高了部分医疗技术服务项目的价格,但调整并不全面系统,仅仅依据药品加成 损失的额度来测算调整幅度,并作为政策损失的补偿,而未与实际劳务价值密切关联。因此, 科 学、合理地调整、规范医疗服务价格势在必行。

\section{3 等级医院之间资源平衡}

各等级医院由于地理位置、人员配置不同, 出现资源不同程度上的不均衡, 也许这样导 致了目前一级医院的次均费用较低, 但是药占比例偏高的局面。

\section{References}

[1] Chun-li ZAHNG, Zhen ZENG. Analysis of personal health expenditure of Zhejiang residents since the new medical reform[J].Health Economic Research,2014(12):42-44

[2] Jin-jun ZHU, An-jue WANG, etc, The analysis of the effect of zero - rate sales on the economic operation of primary medical institutions in Anhui Province[J].Anhui Medicine.2012, 16(10):1556-1558.

[3] Zhen-fang HE, Hong DING, etc, The evaluation of the policy effect of zero difference rate of medicine in county public hospitals in Anhui Province[J].Chinese Health Administration. 2014, (8):590-592.

[4] Zheng-xian YING, Xiao-jun HU, etc, The practice and thinking about the reform of drug zero rate in the people's hospital of Dongyang[J].China Hospital Management Journal[J].2014,30(5):337-339

[5] Qiang YU. Some thoughts on how to deal with commercial bribery of pharmaceutical purchase and sale[J].Hospital Management BBS.2013,30(9):29-31

[6] Yun-liang WU, Wen-ying CHI, etc, Construction and implementation of performance bonus distribution system in public hospitals[J].Health Economic Rsearch. 2014,(9):36-38 